\title{
BMJ Open Understanding and reducing the prescription of hypnotics and sedatives at the interface of hospital care and general practice: a protocol for a mixed-methods study
}

\author{
Stephanie Heinemann, ${ }^{1,2}$ Vivien Weiß, ${ }^{1}$ Kati Straube, ${ }^{1,3}$ Roland Nau, ${ }^{3,4}$ \\ Thomas Grimmsmann, ${ }^{5}$ Wolfgang Himmel, ${ }^{1}$ Eva Hummers-Pradier ${ }^{1}$
}

To cite: Heinemann $\mathrm{S}$, Weiß V, Straube K, et al. Understanding and reducing the prescription of hypnotics and sedatives at the interface of hospital care and general practice: a protocol for a mixed-methods study. BMJ Open 2016;6:e011908. doi:10.1136/bmjopen-2016011908

- Prepublication history and additional material is available. To view please visit the journal (http://dx.doi.org/ 10.1136/bmjopen-2016011908).

SH and VW contributed equally.

Received 14 March 2016 Revised 24 May 2016 Accepted 25 May 2016

CrossMark

For numbered affiliations see end of article.

Correspondence to Professor Wolfgang Himmel; whimmel@gwdg.de

\section{ABSTRACT}

Introduction: Hypnotics and sedatives, especially benzodiazepines and Z-drugs, are frequently prescribed for longer periods than recommended - in spite of potential risks for patients. Any intervention to improve this situation has to take into account the interplay between different actors, interests and needs. The ultimate goal of this study is to develop-together with the professionals involved-ideas for reducing the use of hypnotics and sedatives and then to implement and evaluate adequate interventions in the hospital and at the primary and secondary care interface.

Methods and analysis: The study will take place in a regional hospital in northern Germany and in some general practices in this region. We will collect data from doctors, nurses, patients and a major social health insurer to define the problem from multiple perspectives. These data will be explored and discussed with relevant stakeholders to develop interventions. The interventions will be implemented and, in a final step, evaluated. Both quantitative and qualitative data, including surveys, interviews, chart reviews and secondary analysis of social health insurance data, will be collected to obtain a full understanding of the frequency and the reasons for using hypnotics and sedatives.

Ethics and dissemination: Approval has been granted from the ethics review committee of the University Medical Center Göttingen, Germany. Results will be disseminated to researchers, clinicians and policy makers in peer-reviewed journal articles and conference publications. One or more dissemination events will be held locally during continuous professional development events for local professionals, including (but not confined to) the study participants.

\section{INTRODUCTION}

Hypnotic and sedative drugs, especially benzodiazepines and Z-drugs are frequently

\section{Strengths and limitations of this study}

- This study makes use of a close cooperation between a university department of general practice, a regional general hospital, a number of primary care practices and a large social health insurer to study the use of hypnotics and sedatives in the hospital and at the primary and secondary care interface.

- The mixed-methods design will combine insurance data, patient chart reviews, standardised surveys of patients and health personnel as well as qualitative interviews to analyse hypnotic and sedative prescriptions and to obtain deeper insight into the attitudes and experiences of hospital staff and general practitioners (GPS).

- Interventions for reducing the use of hypnotics and sedatives will be developed in focus groups with all relevant stakeholders to support compliance.

- The success of the interventions will strongly depend on the willingness of the hospital staff and GPs to change familiar and established workflows and implement new strategies in handling patients with sleeping problems.

prescribed and in many cases for longer periods than recommended-in spite of the potential risks for patients such as addiction, falls, cognitive impairment and depressive symptoms. ${ }^{1-4}$ These drugs are often started during an acute situation, for example, during a personal crisis or hospital stay. It seems that in these cases, drugs such as benzodiazepines and Z-drugs are given because of a perceived lack of alternative treatment options $^{5}$ or because physicians regard other medical issues with higher priority than the restriction of hypnotics and sedatives. ${ }^{6}$

In the hospital setting, different professional groups may play a role in the relatively 
high level of hypnotic and sedative prescribing. While doctors are responsible for diagnosis and treatment (ie, prescription of drugs), nurses dispense and document the use of p.r.n. ${ }^{i}$ drugs. The decision of when to administer a p.r.n. drug is generally left to the nurse. Once a drug has been given in the hospital setting, it becomes possible that its use is carried over into primary care. ${ }^{7}$ Such chain reactions between primary and secondary care have been described for other drugs, such as proton pump inhibitors, ${ }^{8} 9$ but not hypnotics and sedatives. We only know from a recently published survey that German general practitioners $(\mathrm{GPs})^{10}$ complain about hospital discharge letters in which sleeping pills are recommended without any need in the patient's home.

To study the knowledge and attitudes of the professionals involved can give insight into the reasons for high benzodiazepine and Z-drug use. Hoffmann ${ }^{11}$ surveyed German GPs about the risks and benefits of these drugs, discovering that Z-drugs are perceived to be more effective and less harmful than benzodiazepines, although there is little evidence to support this. ${ }^{12}$ However, the attitudes of hospital doctors and nurses towards these commonly used drugs are unknown.

Hypnotic and sedative use could also be influenced by patient preferences. Patient satisfaction and (perceived, short-term) improvement of quality of life may motivate prescriptions. Over $90 \%$ of general practice patients taking benzodiazepines reported at least one benefit and $50 \%$ of respondents reported that they 'feel better overall'. ${ }^{13}$ Due to this kind of 'magic bullet' potential of benzodiazepines and Z-drugs, GPs might prescribe them because they feel overwhelmed by the psychosocial problems of their patients. ${ }^{14}$ However, we do not know whether a positive experience in the primary care setting may motivate patients to ask for a sleeping pill in the hospital and, vice versa, whether a positive experience in the hospital may be a reason why patients ask their primary care physician to continue this drug after discharge.

In Germany, it is possible for doctors to prescribe drugs for social health insurance patients via so-called 'private prescriptions'. ${ }^{15}$ In this case, the patient pays the entire cost for the drugs out of pocket. Since social health insurers have no record of these prescriptions, it may be possible that physicians prescribe drugs associated with abuse as private prescriptions to avoid liability issues. Looking at pharmacy data, Hoffmann and Glaeske $^{17}$ found that private prescriptions made up nearly half $(49 \%)$ of all prescriptions for zolpidem, a commonly prescribed Z-drug. However, we know very little about the doctors' reasons and motives for issuing private prescriptions.

'These drugs are labelled 'p.r.n. drugs' (from Latin: 'pro re nata'; meaning 'as needed' or 'as the situation arises').
Any intervention to reduce the prescription and use of hypnotics and sedatives has to take into account this interplay between different actors, interests and needs. Therefore, we will study

- The frequency of hypnotic and sedative use during hospitalisation;

- The continuation or discontinuation of these drugs in primary care;

- The attitudes of hospital doctors and nurses towards hypnotics and sedatives;

- The reasons for beginning hypnotic and sedative prescriptions in primary care and in hospital, including the use of private prescriptions; and

- The experience of hospitalised patients with these drugs.

The ultimate goal of this study is to develop-together with the professionals involved-ideas for reducing the use of hypnotics and sedatives and then to implement and evaluate adequate interventions in the hospital and at the primary and secondary care interface.

\section{METHODS AND ANALYSIS}

This 2-year study makes use of a close cooperation between a university department of general practice, a regional general hospital, a number of primary care practices in the region and a large social health insurer. The framework for designing and evaluating complex interventions to improve healthcare from Campbell et $a l^{18}$ will be used in a supporting manner to guide the research and to develop an intervention. Figure 1 shows
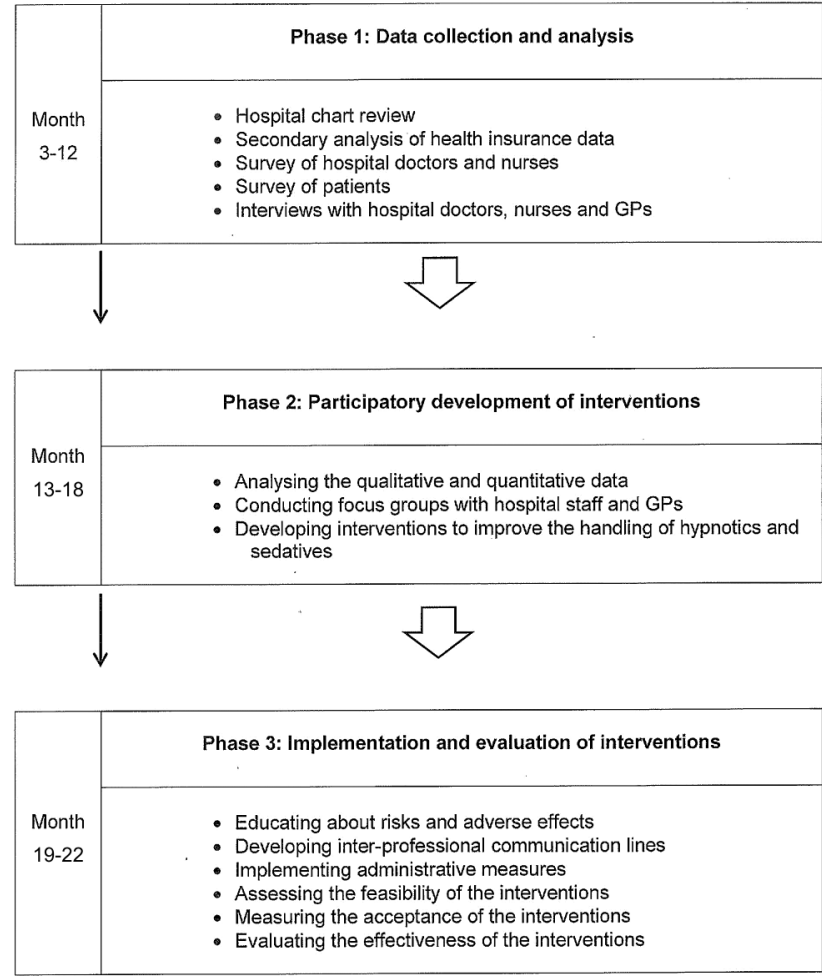

Figure 1 Flow chart of study phases. 
the three phases of the project. First, we will collect data from doctors, nurses, patients and a social health insurer to define the problem from multiple perspectives. Second, we will explore these data with relevant stakeholders to develop ideas for an intervention. Third, the intervention will be implemented and evaluated.

\section{Phase 1: data collection and analysis}

Five different data sources or groups of informants will be addressed, using both quantitative and qualitative methods of data collection and analysis.

\section{Hospital chart review}

Aim: To determine the amount of hypnotics and sedatives administered in a regional hospital and possible patient characteristics associated with administration.

Data source and data collection: This retrospective chart review will include all patients $\geq 65$ years who were hospitalised during a defined period of time. Patients of all wards in the hospital (internal medicine, geriatrics, trauma surgery, general surgery, urology, plastic surgery and otolaryngology) will be included. Patients without at least one overnight stay and cases of death will be excluded. Anonymised data will be collected from the clinical records of the patients using a computer-based form. It will be password-secured and stored on the security servers of the University of Göttingen. The data will include the following information:

- Age and sex of the patient;

- Duration of hospital stay;

- Referral from (home, other hospital, rehabilitation or nursing home);

- Discharge to (home, other hospital, rehabilitation or nursing home);

- Hospital ward;

- Actual diagnosis and other diagnoses;

- Addictive disorders;

- Prescribed and administered hypnotics and sedatives;

- Dosages and number of times hypnotic and sedative drugs were taken;

- Medication on admission;

- Discharge medication.

Data analysis: The absolute and relative number of patients who receive one or more benzodiazepines, Z-drugs, antidepressants and antipsychotics during their hospital stay will be analysed. Predictors for the prescription of hypnotics and sedatives, such as patient age and gender, condition or the hospital ward will be identified by multivariate logistic regression analyses.

\section{Secondary analysis of health insurance data}

Aim: To ascertain the overall influence of hospitalisation on the prescription of anxiolytics and hypnotics and sedatives, especially benzodiazepines and Z-drugs in outpatient care.

Data source and data collection: Our data base comprises primary care prescription data from all patients who (1) live in the federal states of Berlin, Brandenburg and
Mecklenburg-West Pomerania and (2) are insured by one of the largest social health insurers in Germany. We will select all patients who were hospitalised in 2012 and compare their prescriptions before and after hospitalisation. The following data will be available:

- Pseudonymised identification number of the insured person, including age and sex;

- Dates of hospital admission and discharge, including the hospital wards;

- Anatomical Therapeutic Chemical (ATC) classification system code and pack size of each prescription.

As social health insurance claim data in Germany do not contain any information about prescriptions in hospital, the analysis will be restricted to outpatientdispensed prescriptions.

Data analysis: At the patient level, we will compare the number of benzodiazepines and Z-drugs (ATC codes N05BA, N05CD, N05CF) dispensed in the primary care sector before and after hospitalisation. We will define a prescription of benzodiazepines and Z-drugs as a 'new prescription' if a patient has not received such a drug during 50 days before hospitalisation but receives it during the 50 days following a hospitalisation, and as a 'long-term medication' if repeat prescriptions occur in the second 50 days after hospitalisation. These periods of time were chosen because a normal prescription typically comprises a package of up to 50 units (eg, tablets). Statistical analysis will include, besides others, the McNemar test for dependent samples.

\section{Survey of hospital doctors and nurses}

Aim: To understand the knowledge and attitudes of hospital doctors and nurses with regard to hypnotics and sedatives, especially in terms of their risks and benefits.

Data source and data collection: All doctors $(\sim 120)$ and nurses $(\sim 260)$ working in the cooperating general hospital will be invited to participate in the survey. A previously published questionnaire for $\mathrm{GPs}^{19}$ will be adapted to the hospital situation and distributed to all doctors and nurses together with their pay cheques. The study team will promote the study with informative posters and personally invite employees to participate in the study, for example, during routine meetings.

Data analysis: We will compare doctors' and nurses' points of view about the frequency of use, benefits and harms of hypnotics and sedatives, differentiating between benzodiazepines and Z-drugs. In a multivariate logistic regression, we will analyse whether the likelihood of prescribing or dispensing a hypnotic or sedative drug increases according to medical specialty or ward (surgery, internal medicine, geriatrics), professional group (doctors or nurses) and years of experience. A special focus will be given to contrasting the perceptions of hospital doctors and nurses. First, we will contrast each group's perception of how often benzodiazepines and Z-drugs are prescribed (doctors) and dispensed (nurses). Also, we will look in detail at how each 
professional group perceives the benefits and side effects of these drugs.

\section{Survey of patients}

Aim: To compare patients' self-reported use of sleeping pills in the hospital with the data from the hospital records, and to survey patients' experiences of effects and side effects, and their attitudes towards use of these drugs at home.

Data source and data collection: Five hundred inpatients $\geq 65$ years will be personally interviewed using a standardised format 1 day before or on the day of their hospital discharge. Data from consenting patients will be matched with their patient file information on the use of benzodiazepines, Z-drugs, mirtazapine, melperone or other hypnotics and sedatives. Data will be stored and analysed in a pseudonymised form.

Data analysis: Interview responses will be analysed descriptively. To determine possible predictors for the wish to receive sleeping pills also after discharge, age, gender, hospital ward will be included in multivariate models. Agreement between the patients' reported use of hypnotics or sedatives and the hospital file will be determined by the $\kappa$ statistics.

\section{Interviews with hospital doctors, nurses and GPs}

Aim: To understand the usage of hypnotics and sedatives from the prescriber and dispenser perspective-particularly in hospital care-and to reconstruct the decisionmaking and prescribing processes at the primary and secondary care interface. Research questions which guide this part of the study are: Why do hospital doctors, nurses and GPs prescribe/dispense hypnotics and sedatives; what are the trigger and reasons? Which factors influence an increased use of hypnotics and sedatives (eg, high workload, lack of time, ambiguous division of labour, pressure to act)? Since these are rather sensitive topics, they will be addressed in an open and unobtrusive way so that our interview partners feel encouraged to talk about them.

Data source and data collection: The sample of interview partners should comprise 10-12 participants from each occupational group (hospital doctors, nurses, GPs). Hospital doctors and nurses will be recruited from the regional general hospital through word-of-mouth and telephone requests. GPs will be recruited by telephone or through face-to-face contacts. Sampling will consider gender, age, job function and length of experience, aiming at maximum variability. The sample of GPs will also consider the practice location. Semistructured interviews (see online supplementary appendices 1-3 for interview guidelines) will last about $30-45 \mathrm{~min}$. The interviews will be recorded and transcribed.

In accordance with recommended principles for conducting qualitative research, the interviews will begin with a narrative opening question; however, a selfdeveloped topic guide will provide a flexible framework to explore beliefs that were not spontaneously covered in the participants' initial narrative.

The topic guide will be developed on basis of the previous quantitative survey and a literature review. Topics will include experiences and attitudes regarding the prescription and handling of hypnotics and sedatives, external influences such as reimbursement method and physician-patient relations, the possibility of "private prescriptions', knowledge about the benefits and risks of hypnotics and sedatives, alternative therapies for insomnia, critical incidents and requested support.

Data analysis: The interviews will be analysed according to Mayring's ${ }^{19}$ qualitative content analysis. Materials will be coded using an inductive procedure. Categories obtained will be discussed by an interprofessional research team with expertise in hospital geriatrics, family medicine, nursing science, health services research and sociology to validate ratings and achieve consensus.

\section{Phase 2: participatory development of interventions}

To take the complexity of the situation into account, we will invite all relevant stakeholders, especially practitioners at the grass roots level, as partners in the research process. ${ }^{20}$ These partners will join us to discuss and develop interventions with the aim of reducing the prescription and use of hypnotics and sedatives. The most important component of such a participatory approach ${ }^{20}$ will be a series of focus groups with hospital doctors, nurses and GPs to discuss the phase 1 results. Each group will consist of about 10 participants. Each focus group will start with a short feedback on our previously collected quantitative and qualitative data. We will then try to stimulate a discussion about how to change the situation. Ample opportunity will be given to the multiple views of the problem in order to guarantee that the different needs of the participants can be addressed and considered when developing ideas for interventions. We will reflect on the needs and input from the local stakeholders in light of the current state of research about effective interventions for reducing hypnotics and sedatives.

During the sessions, preliminary results will be compiled following by the knowledge mapping method. ${ }^{21} \mathrm{~A}$ final synopsis will be circulated among all members. ${ }^{20}$

\section{Phase 3: implementation and evaluation of interventions}

The final phase of the project will be based on the results of the data collection (phase 1) and the focus group discussions (phase 2) and include the implementation and evaluation of interventions.

\section{Implementation of interventions}

Successful implementation of interventions depends, besides others, on the target audience's willingness to change behaviour. We will have to consider a variety of attitudes and opinions of nurses, hospital doctors and GPs as well as a variety of organisational structures. For 
example, nurses are often the first health professionals to be contacted about sleeping problems in hospitals. In primary care, however, patients typically address their sleep problems directly to their GP. Therefore, it will be necessary to develop interventions which address both the actions and strategies of the individuals involved as well as the organisational structures of their professional working environment.

Interventions may involve the following topics:

- Discussing the risks and adverse effects of hypnotics and sedatives within the interprofessional team, including how to inform patients about risks and possible alternatives, including non-pharmacological strategies;

- Improving interprofessional communication lines in challenging situations and developing a team spirit for balancing patient needs and workplace demands;

- Implementing administrative measures (eg, a standardised care pathway for patients with insomnia, quality management indicators) for handling hypnotics and sedatives.

\section{Evaluation}

The evaluation of the interventions will address two aspects: (1) feasibility and (2) effectiveness, including the following questions:

- Will all target groups accept, and participate in, the interventions?

- Will the participants be satisfied with the interventions?

- Can the interventions reduce the amount of hypnotics and sedatives being prescribed in the hospital and in general practice?

The research team will record and analyse quantitative data about the participation rates of the different professional groups in intervention activities, for example, how many nurses participated, from which wards, etc, in order to measure whether all relevant professional groups could be reached. Participants in intervention activities, for example, a workshop about the treatment of sleeping problems, will be asked to fill in a survey form about their satisfaction with the workshop, its relevance and remaining knowledge gaps regarding hypnotic and sedative drugs.

After the interventions, hospital employees will be asked to self-rate their competence in handling hypnotic and sedative drugs within the framework of a regular employee survey. The hospital pharmacy will provide benchmarking data in terms of the type and amounts of hypnotic and sedative drugs dispensed in the different departments before and after the interventions.

A larger, controlled effectiveness trial of the intervention featuring clinical outcomes is outside the scope of this project and requires additional funding.

\section{DISCUSSION}

Following the framework for designing and evaluating complex interventions, ${ }^{18}$ we will collect data from various sources (see phase 1) to explore the context and structural surrounding conditions that influence the prescription and use of hypnotics and sedatives and then bring together all professional parties involved to develop interventions (see phase 2) that help to avoid unnecessary prescriptions and use of hypnotics and sedatives in primary and secondary healthcare. The evaluation will focus on feasibility and effectiveness of the interventions (see phase 3).

\section{Strengths and limitations}

Due to the close cooperation between a university department and a regional general hospital, this project will have the opportunity to investigate the attitudes and experiences of doctors and nurses concerning hypnotics and sedatives and thus try to reconstruct the prescribing and dispensing process at the primary and secondary care interface. Other studies have concentrated on individual actors of the primary and secondary care interface separately, ${ }^{11}{ }^{13}{ }^{22}$ whereas we will look at the interaction between professional groups, patients and settings and include all wards. This design will contribute to a comprehensive description of the problem.

To measure the frequency of hypnotics and sedatives, we will use different data sources and different methods, namely the combination of a hospital chart review and a secondary analysis of social health insurance data. This design will contribute to the validity of our data about the use of hypnotics and sedatives in the hospital and at the primary and secondary interface. Our research will be dependent on well-documented hospital charts and social health insurance records. The analysis of continuation or discontinuation of these drugs on the interface between the hospital and primary care will be dependent on the completeness of discharge letters.

Using the hospital charts, it is, on principle, not possible to exactly determine whether the drugs under study have been administered because of sleeping problems or other reasons. We only know from exploratory discussions with experts in the hospital that the majority of hypnotics and sedatives are described for sleeping problems. Moreover, we will compare the patients' answers about the drugs used in the hospital with the data in the hospital charts. In case of a high agreement, we can conclude that the drugs under study were, indeed, used for sleeping problems.

With regard to the recruitment of interviewees, we expect a selection bias: individuals who are sensitive to the problems and risks associated with hypnotics and sedatives will be more likely to participate. Furthermore, it could be possible that we obtain socially desirable answers in both surveys as well as semistructured interviews.

For the patient survey, we will not consider patients who suffer from severe forms of dementia as interview partners; consequently, we cannot explore the perspectives of these patients, who are often treated with hypnotics and sedatives. 
In terms of a sustainable development, it would be desirable to extend the interprofessional intervention created here to other hospitals, family practices and/or quality circles. A further aim will be to develop a larger trial to evaluate the effectiveness of an interprofessional intervention for reducing hypnotic and sedative use in hospitals and/or primary care.

\section{ETHICS AND DISSEMINATION}

Patient and staff information sheets will be distributed on the wards and clinical areas before and during the study. Before the study, the researchers will spend time in each ward to make the staff aware of the study, respond to any queries about the study and hand them information sheets. All participants will receive written information sheets to provide written informed consent. All person-related data will be collected and treated according to current data privacy legislation. Medical and nursing staff as well as GPs and patients participating in the study will be assigned a unique participant identifier. Clinical records included will be also assigned a unique participant identifier. The list of clinical records included, participant identifier and associated identification numbers will be kept separate from the data collection in the university department. This data key list will be destroyed at the end of the study.

Results will be disseminated among researchers, clinicians, medicals schools, nursing schools and health planners in peer-reviewed journal articles and conference publications. Additionally, the findings of the research in the hospital setting will be reported in the hospital staff magazine. Local events for continuous professional development will share the research results among local professionals, including (but not confined to) the study participants.

\section{Author affiliations \\ ${ }^{1}$ Department of General Practice, University Medical Center, Göttingen, Germany}

${ }^{2}$ Department of Health Sciences, University of Applied Sciences, Fulda, Germany

${ }^{3}$ Department of Geriatrics, Evangelisches Krankenhaus Göttingen-Weende, Göttingen, Germany

${ }^{4}$ Institute of Neuropathology, University Medical Center, Göttingen, Germany ${ }^{5}$ German Health Insurance Medical Service (MDK) Mecklenburg-Vorpommern, Schwerin, Germany

Contributors All authors contributed to the design of the research. EH-P, RN and $\mathrm{WH}$ are the initiators and primary supervisors of the project. SH and VW are two of the main researchers and drafted the manuscript. KS is responsible for the chart review; TG is responsible for the secondary analysis of the health insurance data. All authors contributed to the manuscript and approved the final version

Funding The study is funded by the German Federal Ministry of Health; the research grant was awarded in a competitive, peer-reviewed procedure (grant number: FKZ-IIA5-2513DSM228).

Competing interests None declared.

Ethics approval Göttingen University Medical Center Ethics Committee (ref number 25/2/14)

Provenance and peer review Not commissioned; externally peer reviewed.
Open Access This is an Open Access article distributed in accordance with the Creative Commons Attribution Non Commercial (CC BY-NC 4.0) license, which permits others to distribute, remix, adapt, build upon this work noncommercially, and license their derivative works on different terms, provided the original work is properly cited and the use is non-commercial. See: http:// creativecommons.org/licenses/by-nc/4.0/

\section{REFERENCES}

1. Jahnsen K, Roser P, Hoffmann K. The problems of long-term treatment with benzodiazepines and related substances: prescribing practice, epidemiology and the treatment of withdrawal. Deutsches Ärzteblatt 2015;112:1-7.

2. Olfson M, King M, Schoenbaum M. Benzodiazepine Use in the United States. JAMA Psychiatry 2015;72:136-42.

3. Schubert I, Küpper-Nybelen J, Ihle $\mathrm{P}$, et al. Prescribing potentially inappropriate medication (PIM) in Germany's elderly as indicated by the PRISCUS list. An analysis based on regional claims data. Pharmacoepidemiol Drug Saf 2013;22:719-27.

4. National Institute for Health and Care Excellence (NICE). Guidance on the use of zaleplon, zolpidem and zopiclone for the short-term management of insomnia. 2004 https://www.nice.org.uk/guidance/ ta77/chapter/about-this-guidance (accessed 16 Feb 2016).

5. Sirdifield C, Anthierens S, Creupelandt H, et al. General practitioners experiences and perceptions of benzodiazepine prescribing: systematic review and meta-synthesis. BMC Fam Pract 2013;14:191.

6. Cook JM, Marshall R, Masci C, et al. physicians' perspectives on prescribing benzodiazepines for older adults: a qualitative study. J Gen Intern Med 2007;22:303-7.

7. Warie H, Petrovic M, Somers A, et al. The use of hypnosedative drugs in a university hospital setting. Acta Clin Belg 2003;58:225-32.

8. Grimmsmann T, Schwabe U, Himmel W. The influence of hospitalisation on drug prescription in primary care-a large-scale follow-up study. Eur J Clin Pharmacol 2007;63:783-90.

9. Himmel W, Kochen MM, Sorns U, et al. Drug changes at the interface between primary and secondary care. Int J Clin Pharmacol Ther 2004:42:103-9.

10. Adam $H$, Niebling WB, Schott $G$. The information about discharge medication: what do general practitioners need? Dtsch Med Wochenschr 2015;140:e74-9.

11. Hoffmann F. Perceptions of German GPs on benefits and risks of benzodiazepines and Z-drugs. Swiss Med Wkly 2013;143:w13745

12. Gunja N. In the Zzz zone: the effects of Z-drugs on human performance and driving. J Med Toxicol 2013;9:163-71.

13. Siriwardena AN, Qureshi MZ, Dyas JV, et al. Magic bullets for insomnia? Patients' use and experiences of newer ( $Z$ drugs) versus older (benzodiazepine) hypnotics for sleep problems in primary care. Br J Gen Pract 2008;58:417-22.

14. Anthierens $\mathrm{S}$, Habraken $\mathrm{H}$, Petrovic $\mathrm{M}$, et al. First benzodiazepine prescriptions. Can Fam Physician 2007(53):1200-5.

15. Weiß V, Hauswaldt J. Off-record prescription of hypnotics. Analysis from family physicians' electronic health records [in German]. Z Allg Med 2015;91:408-12.

16. Hoffmann F, Hies M, Glaeske G. Regional variations of private prescriptions for the non-benzodiazepine hypnotics zolpidem and zopiclone in Germany. Pharmacoepidemiol Drug Safe 2010;19:1071-7.

17. Hoffmann F, Glaeske G. Benzodiazepine hypnotics, zolpidem and zopiclone on private prescriptions: use between 1993 and 2012 [in German]. Nervenarzt 2014;85:1402-9.

18. Campbell NC, Murray E, Darbyshire J, et al. Designing and evaluation complex interventions to improve health care. BMJ 2007;334:455-9.

19. Mayring P. Qualitative content analysis: theoretical foundation, basic procedures and software solution. 2014 http://www.psychopen.eu/ fileadmin/user_upload/books/mayring/ssoar-2014-mayringQualitative content analysis theoretical foundation.pdf (accessed 17 Feb 2016)

20. Craig $P$, Dieppe $P$, Macintyre $S$, et al. Developing and evaluating complex interventions: the new Medical Research Council guidance. Int J Nurs Stud 2013;50:587-92.

21. Pelz C, Schmitt A, Schmitt M. Knowledge mapping as a tool for analyzing focus groups and presenting their results in market and evaluation research [in German]. Forum Qual Soc Res 2014;5:2.

22. Anthierens $\mathrm{S}$, Habraken $\mathrm{H}$, Petrovic $\mathrm{M}$, et al. The lesser evil? Initiating a benzodiazepine prescription in general practice: a qualitative study on GPs' perspectives. Scand J Prim Health Care 2007;25:214-19. 